\title{
Comparative analysis of an mcr-4 Salmonella enterica subsp. enterica monophasic variant of human and animal origin
}

\author{
Alessandra Carattoli ${ }^{1}$, Edoardo Carretto ${ }^{2}$, Flavia Brovarone ${ }^{2}$, Mario Sarti $^{3}$ and Laura Villa ${ }^{1 *}$ \\ ${ }^{1}$ Department of Infectious Diseases, Istituto Superiore di Sanità, Rome, Italy; ${ }^{2}$ Clinical Microbiology Laboratory, IRCCS Arcispedale \\ Santa Maria Nuova, Reggio Emilia, Italy; ${ }^{3}$ Clinical Microbiology Laboratory, S. Agostino-Estense Hospital, Baggiovara, Italy \\ ${ }^{*}$ Corresponding author. E-mail: laura.villa@iss.it
}

Received 12 March 2018; returned 10 April 2018; revised 26 July 2018; accepted 31 July 2018

\begin{abstract}
Objectives: In this study we compared the recently described mcr-4-positive Salmonella enterica monophasic variant, isolated in 2016 in two Italian patients affected by gastroenteritis, with the first mcr-4-positive Salmonella isolate identified in 2013 in a pig at slaughter in Italy.

Methods: WGS of the two Salmonella isolates of human origin was performed using a MiSeq instrument (Illumina). The phylogenetic analysis was performed by SNP analysis, comparing genomes of the mcr-4-positive isolates of swine and human origin with 82 Salmonella genomes downloaded from the EnteroBase Salmonella database. Complete sequences of plasmids carrying mcr-4.2 were obtained and compared. Transformation experiments were performed to transfer the mcr-4 plasmids into a colistin-susceptible Escherichia coli recipient strain.
\end{abstract}

\begin{abstract}
Results: Comparative genomics demonstrated that the Salmonella of swine origin did not cluster with the isolates of human origin. The mcr-4.2 gene variant identified in the Salmonella of human origin was located on a ColE-like plasmid. This plasmid showed different replication and mobilization genes with respect to those previously described in the ColE plasmid carrying the mcr-4.1 variant, identified in Salmonella of swine origin.
\end{abstract}

Conclusions: The divergence in genomes, plasmids and gene variants demonstrated that there was not a unique mcr-4-positive, monophasic Salmonella lineage circulating in animals and causing gastroenteritis in humans in Italy. There was no horizontal transfer of the same plasmid among Salmonella strains of animal and human origin, but the mcr-4 gene and a fragment of the plasmid identified in the animal strain were mobilized by an IS1294 into a different ColE plasmid.

\section{Introduction}

Colistin is a decades-old drug, intensively used in veterinary medicine. In humans, the global spread of MDR Gram-negative bacteria with limited therapeutic options resulted in the return of colistin use, despite its documented toxicity. ${ }^{1}$ Two major mechanisms of colistin resistance have been described in Gram-negative bacteria: chromosomal mutations occurring in genes involved in the synthesis and modification of LPS and the acquisition of mobile colistin resistance $(\mathrm{mcr})$ genes encoding phosphoethanolamine transferases, resulting in a more cationic charged LPS that confers reduced susceptibility to colistin. ${ }^{2}$ To date, five types of $\mathrm{mcr}$ genes have been described. mcr-1 was the first to be reported and retrospective analysis on different bacterial collections demonstrated worldwide spread of the mcr-1 gene. ${ }^{3,4}$ The $\mathrm{mcr}-2$, mcr-3, mcr-4 and $\mathrm{mcr}-5$ genes were more recently discovered and their distribution and prevalence are still under investigation. ${ }^{5-8}$ The mcr-4.1 gene was first described in an Italian strain that was a Salmonella enterica subsp. enterica monophasic variant of serovar Typhimurium with antigenic formula 4,[5],12,i:- isolated from a pig slaughtered in Italy in 2013 (Salmonella strain R3445). ${ }^{7}$ Recently, mcr-4.2-positive Salmonella serovar 4,[5],12,i:- strains were reported from two apparently unrelated human gastroenteritis cases occurring in Italy in $2016 .{ }^{9}$ In this study the analysis of the plasmid carrying the mcr-4.2 gene and the genomic comparison of Salmonella strains of human and animal origin was performed, with the aim to better describe characteristics, similarities and differences among the mcr-4-positive strains from Italy.

\section{Materials and methods}

\section{Bacterial strains}

Two S. enterica with antigenic formula 4,[5],12:i:- were isolated from faecal samples of two Italian patients with gastroenteritis, collected in October 2016 (strain AB-160) and November 2016 (strain AB-243), at S. AgostinoEstense Hospital. ${ }^{9}$ The R3445 S. enterica monophasic variant 4,[5],12:i:- 


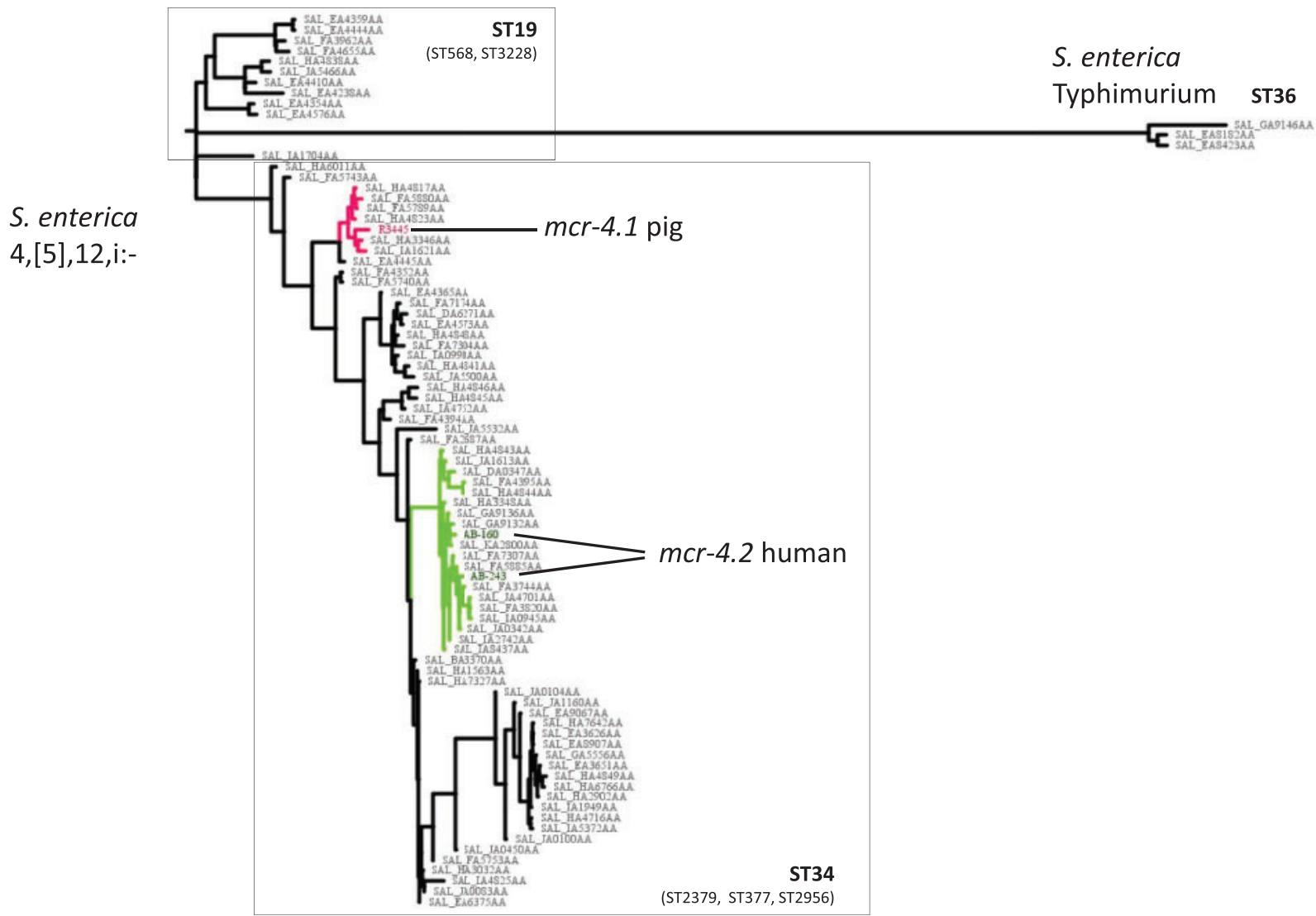

Figure 1. Phylogenetic tree of human and animal mcr-4-positive Salmonella genomes. Phylogenetic maximum-likelihood tree generated using FigTree v1.4.3 software of the SNP analysis performed using the KSNP3 tool. The clades of the Salmonella of pig (R3445) and human (AB-160 and AB-243) origin are highlighted in bold. The labels of the branches correspond to the Uberstrain codes as they appear in the EnteroBase Salmonella database. Three genomes (SAL_GA9146AA, SAL EA8182AA and SAL EA8423AA) were from S. enterica serovar Typhimurium; all the others were S. enterica monophasic variants. The branches generated by genomes assigned to ST34 or to ST19 and their related STs (differing from ST34 and ST19 by one single allele of the MLST profile, respectively) are boxed. This figure appears in colour in the online version of JAC and in black and white in the print version of JAC.

was identified in a pig at slaughter in Italy in $2013 .^{7}$ Colistin MICs for Salmonella strains and their respective $\mathrm{mcr}$-4-positive transformants were determined using a broth microdilution (BMD)-based commercial system (SensiTest Colistin, Liofilchem, Italy) and then confirmed using the BMD method according to CLSI and EUCAST (ISO standard method 20776-1). ${ }^{10-12}$ All the isolates were tested in triplicate. Three reference strains (Escherichia coli ATCC 25922, Pseudomonas aeruginosa ATCC 27853 and E. coli NCTC 13846) were used as controls.

\section{Transfer of pMCR4 plasmids}

Plasmid DNA was purified from strains $A B-160$ and $A B-243$ with the Plasmid Mini Kit (QIAGEN) and used to transform E. coli DH5 $\alpha$ chemically competent cells (Invitrogen). Transformants were selected on LB agar plates (Sigma) containing colistin $(2 \mathrm{mg} / \mathrm{L}$ ) and the colonies were tested by PCRs using mcr-4-specific primers. ${ }^{7}$ Transformants of pMCR-4.1_R3445 in the $E$. coli $\mathrm{DH} 5 \alpha$ recipient were previously obtained and used for comparative analysis. ${ }^{7}$ MICs conferred by the different plasmids to the isogenic E. coli $\mathrm{DH} 5$ a recipient were determined as above.

\section{WGS}

WGS was performed on DNA extracted from the Salmonella strains using the Macherey Nagel Kit. Genomic DNA paired-end libraries were generated using the Nextera XT DNA Sample Preparation Kit (Illumina) and sequenced using a MiSeq instrument with the $2 \times 300$ PE protocol (Illumina). De novo assembly of Illumina reads was performed using the Galaxy version 20150522 of the SPAdes pipeline through the ARIES public Galaxy server (https://w3.iss.it/site/aries/). Draft genome sequences were annotated using the RAST server (http://rast.nmpdr.org/). Antimicrobial resistance, replicon genes and MLST STs were detected using the ResFinder, PlasmidFinder and MLST online tools at the Center for Genomic Epidemiology (CGE) of the Danish Technical University (https://cge.cbs.dtu.dk/services/), respectively. IS elements were detected using ISFinder (https://www-is.biotoul.fr/).

Seventy-nine genomes were selected and downloaded from the EnteroBase Salmonella database (http://enterobase.warwick.ac.uk/), using '4,[5],12,i:-' as the keyword. For comparison, three Salmonella Typhimurium genomes were also downloaded from the EnteroBase Salmonella database and included in an SNP analysis, performed using the kSNP3 tool through the ARIES public Galaxy server. A phylogenetic maximum-likelihood tree was generated using FigTree v1.4.3 software.

The mcr-4 and replicon genes were identified on the same contig in assembled genomes, determining in silico the mcr-4 gene location on plasmids. The complete, circularly closed plasmid was obtained by PCR-based gap closure and sequencing of the amplicon. Purified plasmid DNA was extracted (PureLink HiPure Plasmid Filter Midiprep Kit, Invitrogen) from transformants and the expected plasmid size was verified by restriction with SalI. SalI was chosen to linearize the plasmid since one single 


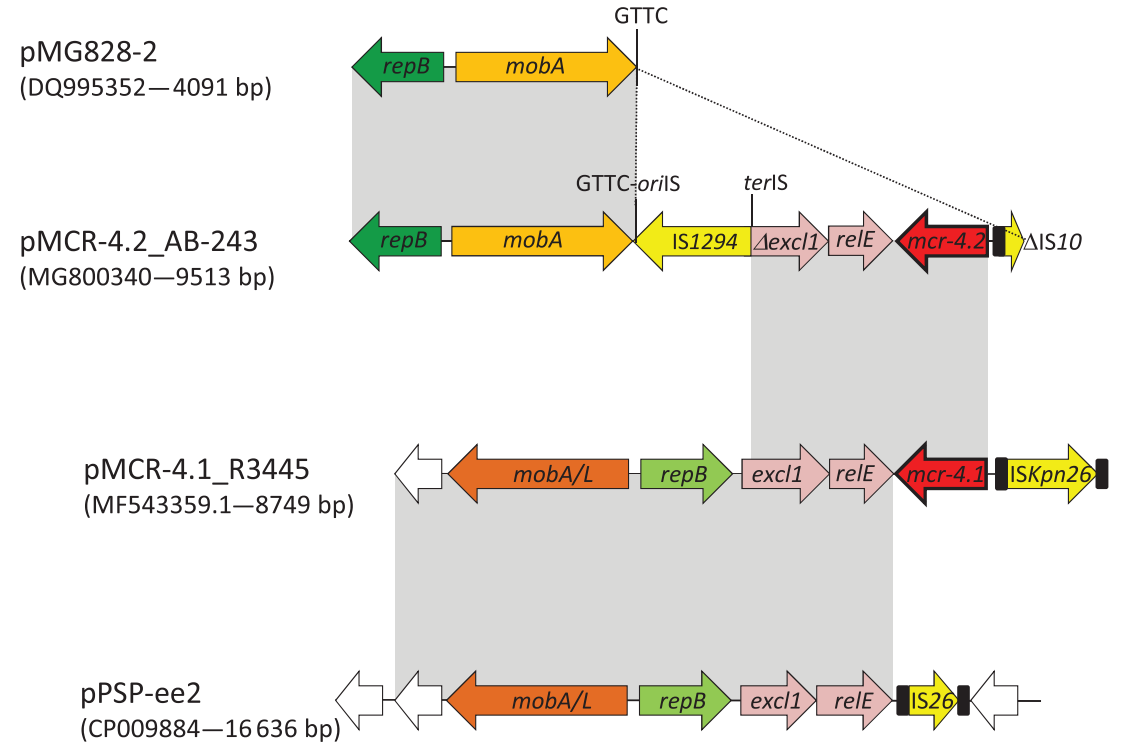

Figure 2. Comparative linear maps of pMCR-4.2_AB-243 and pMCR-4.1_R3445 plasmids and their close relatives. pMCR-4.2_AB-243 and pMCR4.1_R3445 plasmid maps were drawn in comparison with the pMG828-2 and pPSP-ee2 reference plasmid maps. The grey shading indicates common regions among plasmids (at nucleotide identity $>98 \%$ ). Predicted coding sequences are indicated by arrows orientated in the direction of transcription of each respective gene; the sizes of the arrows are not to scale. The IS1294 tetranucleotide (GTTC) sequence-specific binding site is indicated. The oriIS and terIS sequences flanking IS1294 are also indicated. IRs flanking the other IS elements are indicated by black boxes. The sequence of the pMCR-4.2_AB-243 plasmid is available at NCBI GenBank under accession number MG800340. This figure appears in colour in the online version of JAC and in black and white in the print version of JAC.

restriction site was expected from the in silico analysis of the plasmid (data not shown).

\section{Results and discussion}

\section{Phylogenetic analysis of the Salmonella isolates}

MLST STs deduced from WGS revealed that the Salmonella strains of animal (R3445) and human (AB-160 and AB-243) origin all belonged to ST34. The three strains also showed the same antigenic formula, being monophasic variants of serovar Typhimurium 4,[5],12,i:-.

Comparative genome analysis of the three $\mathrm{mcr}$-4-positive Salmonella isolates of Italian origin was performed against a selection of 82 genomes of Salmonella, 3 serovar Typhimurium and 79 monophasic variant 4,[5],12,i:- of human, animal and environmental origin, isolated in Europe, Asia and North America and showing ST36, ST34, ST19, ST2379, ST3377, ST2956, ST3228 and ST568, downloaded from the EnteroBase Salmonella database. The phylogenetic tree showed that $A B-160$ and $A B-243$ strains of human origin were on closely related clades, which were distant from that of the R3445 genome from the pig (Figure 1).

\section{mcr-4 plasmids}

In the AB-243 genome, the ResFinder tool detected the mcr-4.2 gene variant on a single contig of $9568 \mathrm{bp}$ with overlapping extremities, which was also identified by the Col8282 PlasmidFinder probe. The presumptive plasmid was circularized by PCR-based gap closure, obtaining the complete sequence of a ColE-like plasmid of 9513 bp, here named pMCR-4.2_AB-243 (accession number MG800340). In the $A B-160$ genome a plasmid identical to pMCR-4.2 AB-243 was identified. PMCR-4.2 AB-243 carried repB and mobA genes (nt positions 5462 to 9513) highly similar to those of plasmid pMG828-2 (accession number DQ995352). In pMCR-4.2 AB-243, a truncated IS10 (nt positions 4975 to 5465) and an entire IS1294 (nt positions 34 to 1721) were identified, which were not present in pMCR-4.1_R3445 (accession number MF543359.1), the ColE plasmid previously described in the Salmonella of swine origin. ${ }^{7}$ However, upstream of the IS1294 element, a common region (nt positions 1719 to 4974) was shared between pMCR-4.2_AB-243 and pMCR-4.1_R3445, including the mcr-4 gene, the ex $\overline{C l} 1$ and relE (belonging to the RelE/ParE toxinantitoxin family) genes (Figure 2). The comparative analysis of pMG828-2 and PMCR-4.2_AB-243 suggested that excl1, relE, $\mathrm{mcr}$ 4.2 genes and the IS10 fragment were mobilized by IS1294 and integrated into a ColE plasmid highly similar to PMG828-2, in a tetranucleotide GTTC site, known to be one of the IS1294 preferred target sites (Figure 2). ${ }^{13}$

The pMCR-4.2_AB-243 backbone differed from that of pMCR4.1_R3445. The latter was positive for the ColE10 PlasmidFinder probe and carried the mcr-4.1 gene variant flanked by an ISKpn26 element (Figure 2). The mobA/L, repB and excl1 genes of pMCR4.1_R3445 showed 99\% nucleotide identity to the pPSP-ee2 plasmid of the Pantoea sp. PSNIH1 isolate. ${ }^{7}$ Nucleotide identity between PMCR-4.1_R3445 and PPSP-ee2 plasmids ended at 214 bp from the $\mathrm{mcr}-4.1$ stop codon because of an IS26 integration occurring in pPSP-ee2 at this site (Figure 2).

\section{Colistin MIC conferred by the PMCR-4.1 and PMCR-4.2 plasmids}

The two mcr-4-positive plasmids conferred the same colistin MIC of $4 \mathrm{mg} / \mathrm{L}$ when transferred by transformation into the isogenic 
E. coli $\mathrm{DH} 5 \alpha$ strain. The colistin MIC for the recipient E. coli $\mathrm{DH} 5 \alpha$ was $0.5 \mathrm{mg} / \mathrm{L}$, while the colistin MIC for the Salmonella from human or animal sources was $8 \mathrm{mg} / \mathrm{L}$. The mutation of the $\mathrm{mcr}-$ 4.1 into the $\mathrm{mcr}-4.2$ variant and its transfer into a novel ColE-type gave no apparently measurable MIC difference.

\section{Conclusions}

Since the isolation of R3445 in 2013, other additional variants of the $\mathrm{mcr}-4.1$ gene have been described. ${ }^{9,14}$ The $\mathrm{mcr}-4.2$ variant was initially described in Salmonella from human gastroenteritis, but is not exclusive to human isolates since it was also found in a collection of E. coli and Salmonella of animal origin. ${ }^{14}$ However, among the Salmonella isolates from Italy, the $\mathrm{mcr}-4$ gene variants were different in strains of human and animal origin, being $\mathrm{mcr}$ 4.2 and $\mathrm{mcr}-4.1$, respectively. ${ }^{7}$ Moreover, genomic analysis demonstrated that the monophasic Salmonella of human origin did not belong to the same cluster as that of animal origin. Currently, all mcr-4 gene variants seem to be preferentially carried by ColElike plasmids in both E. coli and Salmonella species, even though the ColE plasmids found in human isolates were different from that previously reported in the Salmonella of animal origin. ${ }^{7}$

As demonstrated by the wide distribution of $\mathrm{mcr}-1$ worldwide, it could be hypothesized that $\mathrm{mcr}-4$ has also been spreading undetected among Enterobacteriaceae, with Salmonella representing a hidden reservoir. ${ }^{2}$ The source of the $\mathrm{mcr}$-4-positive Salmonella infecting humans in 2016 in Italy is still unknown. Further epidemiological analyses at the local and national level may be useful to understand the extent of the circulation of this gene in the different sources.

\section{Funding}

This work was supported by the Ministry of Health of Italy (grant number RF-2011-02346987).

\section{Transparency declarations}

None to declare.

\section{References}

1 Landman D, Georgescu C, Martin DA et al. Polymyxins revisited. Clin Microbiol Rev 2008; 21: 449-65.
2 Poirel L, Jayol A, Nordmann P. Polymyxins: antibacterial activity, susceptibility testing, and resistance mechanisms encoded by plasmids or chromosomes. Clin Microbiol Rev 2017; 30: 557-96.

3 Liu YY, Wang Y, Walsh TR et al. Emergence of plasmid-mediated colistin resistance mechanism MCR-1 in animals and human beings in China: a microbiological and molecular biological study. Lancet Infect Dis 2016; 16: 161-8.

4 Schwarz S, Johnson AP. Transferable resistance to colistin: a new but old threat. J Antimicrob Chemother 2016; 71: 2066-70.

5 Xavier BB, Lammens C, Ruhal R et al. Identification of a novel plasmidmediated colistin-resistance gene, $\mathrm{mcr}$-2, in Escherichia coli, Belgium, June 2016. Euro Surveill 2016; 21: $\mathrm{pii}=30280$.

6 Yin W, Li H, Shen Y et al. Novel plasmid-mediated colistin resistance gene mcr-3 in Escherichia coli. mBio 2017; 8: e00543-17.

7 Carattoli A, Villa L, Feudi C et al. Novel plasmid-mediated colistin resistance mcr-4 gene in Salmonella and Escherichia coli, Italy 2013, Spain and Belgium, 2015 to 2016. Euro Surveill 2017; 22: pii $=30589$.

8 Borowiak M, Fischer J, Hammerl JA et al. Identification of a novel transposon-associated phosphoethanolamine transferase gene, $\mathrm{mcr}$ 5 , conferring colistin resistance in $d$-tartrate fermenting Salmonella enterica subsp. enterica serovar Paratyphi B. J Antimicrob Chemother 2017; 72: 3317-24.

9 Carretto E, Brovarone F, Nardini $P$ et al. Detection of $m c r-4$ positive Salmonella enterica serovar Typhimurium in clinical isolates of human origin, Italy, October to November 2016. Euro Surveill 2018; 23: pii=17-00821.

10 Carretto E, Brovarone F, Russello G et al. Clinical validation of SensiTest Colistin, a broth microdilution-based method to evaluate colistin MICs. J Clin Microbiol 2018; 56: e0152317.

11 Clinical and Laboratory Standards Institute. Performance Standards for Antimicrobial Susceptibility Testing: Twenty-Seventh Informational Supplement M100-S27. CLSI, Wayne, PA, USA, 2017.

12 International Organization for Standardization (ISO). ISO 20776-1:2006 Clinical Laboratory Testing and In Vitro Diagnostic Test Systems-Susceptibility Testing of Infectious Agents and Evaluation of Performance of Antimicrobial Susceptibility Test Devices_-Part 1: Reference Method for Testing the In Vitro Activity of Antimicrobial Agents Against Rapidly Growing Aerobic Bacteria Involved In Infectious Diseases. Geneva, Switzerland: ISO, 2006. https://www. iso.org/standard/41630.html.

13 Tavakoli N, Comanducci A, Dodd HM et al. IS1294, a DNA element that transposes by RC transposition. Plasmid 2000; 44: 66-84.

14 Rebelo AR, Bortolaia V, Kjeldgaard JS et al. Multiplex PCR for detection of plasmid-mediated colistin resistance determinants, $\mathrm{mcr}-1, \mathrm{mcr}-2, \mathrm{mcr}-3$, mcr-4 and mcr-5 for surveillance purposes. Euro Surveill 2018; 23: pii $=17-00672$. 